ESAIM: PROCEEDINGS, October 2011, Vol. 33, p. 22-35

C. Dobrzynski, T. Colin \& R. Abgrall, Editors

\title{
MULTISCALE EXPANSION AND NUMERICAL APPROXIMATION FOR SURFACE DEFECTS *
}

\author{
V. Bonnaillie-Nö̈l ${ }^{1}$, D. Brancherie ${ }^{2}$, M. Dambrine $^{3}$, F. Hérau $^{4}$, S. Tordeux $^{5}$ \\ AND G. VIAL ${ }^{6}$
}

\begin{abstract}
This paper is a survey of articles $[5,6,8,9,13,17,18]$. We are interested in the influence of small geometrical perturbations on the solution of elliptic problems. The cases of a single inclusion or several well-separated inclusions have been deeply studied. We recall here techniques to construct an asymptotic expansion. Then we consider moderately close inclusions, i.e. the distance between the inclusions tends to zero more slowly than their characteristic size. We provide a complete asymptotic description of the solution of the Laplace equation. We also present numerical simulations based on the multiscale superposition method derived from the first order expansion (cf [9]).

We give an application of theses techniques in linear elasticity to predict the behavior till rupture of materials with microdefects (cf [6]). We explain how some mathematical questions about the loss of coercivity arise from the computation of the profiles appearing in the expansion (cf [8]).
\end{abstract}

Résumé. Nous faisons ici une synthèse des articles $[5,6,8,9,13,17,18]$. On s'intéresse à l'influence de petites perturbations géométriques sur la solution de problèmes elliptiques. Les cas d'une inclusion isolée ou de plusieurs bien séparées ont été largement étudiés. Nous considérons plus précisément le cas où la distance entre deux inclusions tend vers zéro mais reste grande par rapport à leur taille caractéristique. Nous donnons un développement asymptotique multi-échelle complet de la solution de l'équation de Laplace dans la situation de deux inclusions. Nous présentons également quelques simulations numériques basées sur une méthode de superposition multi-échelle provenant du développement au premier ordre (cf [9]).

Nous étendons ces techniques aux équations de l'élasticité linéaire afin de prédire le comportement à rupture de certains matériaux présentant des micro-défauts (cf [6]). Nous verrons également comment le calcul numérique des profils intervenant dans le développement asymptotique soulève des questions mathématiques liées à la perte de coercivité des problèmes approchés (cf [8]).

* This work has been supported by the ANR project MACADAM $n^{\circ}$ JCJC06-139561. Is has been partially realized during the stay of some of the authors at the CIRM in February 2008 and the authors are grateful for the very good working conditions.

1 IRMAR - UMR6625, ENS Cachan Bretagne, Univ. Rennes 1, CNRS, UEB, av Robert Schuman, 35170 Bruz, France, virginie.bonnaillie@bretagne.ens-cachan.fr

${ }^{2}$ Laboratoire Roberval - UMR6253, Université de Technologie de Compiègne, rue Personne de Roberval, BP 20529, 60205 Compiègne Cedex, France, delphine.brancherie@utc.fr

3 LMAP - UMR5142, Université de Pau et des Pays de l'Adour, av de l'Université, BP 1155, 64013 Pau Cedex, France, marc.dambrine@univ-pau.fr

${ }^{4}$ Laboratoire de mathématiques Jean Leray - UMR6629, Université de Nantes, 2 rue de la Houssinière, BP 92208,44322 Nantes Cedex 3, France, frederic.herau@univ-nantes.fr

${ }^{5}$ INRIA - IPRA, Université de Pau et des Pays de l'Adour, av de l'Université, BP 1155, 64013 Pau Cedex, France, sebastien.tordeux@insa-toulouse.fr

${ }^{6}$ Université de Lyon - CNRS UMR 5208, École Centrale de Lyon, Institut Camille Jordan, 36 avenue Guy de Collongue, 69134 Ecully Cedex, France, gregory.vial@ec-lyon.fr

(C) EDP Sciences, SMAI 2011 


\section{INTRODUCTION}

In many physical situations, one has to consider objects whose geometry involves different scales. Typically, to the macroscopic description should be added a microscopic level of details: this is the case for granulates inside concrete, or bumps on a shell. The questions we are interested in deal in particular with mechanical properties of such materials. The mathematical modelling of these situations consists usually of a system of partial differential equations posed in a (2D or 3D) domain standing for the real geometry. While the theoretical aspects are usually unaffected by microscopic inhomogeneities, the numerics need special care. Indeed, taking into account two different scales in a finite element code requires an adapted mesh refinement in the vicinity of the defects. Resulting computations can become prohibitively costly. Hence, usually, only the macroscopic description is preserved. The influence of local inhomogeneities on the global behavior of the material is then ignored. We aim at designing a numerical method involving the two geometric scales, with a reasonable computational cost.

Our approach is based on a precise asymptotic analysis of the state equation with respect to the characteristic size $\varepsilon$ of the microdefects. The limit solution as $\varepsilon$ tends to 0 corresponds to the solution in the unperturbed domain, which can be reasonably approximated with a coarse mesh. In the framework of our project, the perturbation resulting from the microdefects essentially concentrates near the latters. In a model case, it has been shown that the first corrector consists of a profile, i.e. a function defined on an infinite dimensionless domain, arising in the rapid variable $x / \varepsilon$, that is the scale of the perturbation. This structure suggests a numerical method based on a superposition of the unperturbed solution and the profile. The main difficulties concern the practical computation of the profiles, and the numerical analysis of the algorithmic efficiency. We propose several methods for this computation.

We derive and justify asymptotic expansions in various situations, especially to explain the influence of small inclusions close to each other. These results generalize to linear elasticity in the framework of damage structures to predict the behavior till rupture of materials with two microdefects close to each other. The multiscale superposition method is used in the preliminary step of crack detection, a continuum-discrete damage model is involved for its propagation.

The question of domains with small inclusions or inhomogeneities has been widely studied, especially in the case of electromagnetics and inverse problem, see for example $[1,2,4,11,15,20,24,25]$.

\section{Organization of the paper}

In Section 1, we deal with theoretical results about the asymptotic expansion for the Laplace equation in a domain with small perturbations. Then we apply these results to the Navier equation in the context of fracture mechanics in Section 2. In Section 3, we use the asymptotic expansion to compute the solution of these problems: it suffices to compute the solution of the non-perturbed problem and some profiles (solutions of some exterior problem) to construct a suitable approximation of the solution of the perturbed problem. We perform some simulations for the Laplace equation and for the linear elasticity problem. We also discuss about the numerical approximation of the profiles by introducing artificial boundary conditions.

\section{Asymptotic EXPANSION FOR The LAPLACE EQUATION}

\subsection{The well-known case of one inclusion}

The case of a single inclusion $\omega$ being either in $\Omega_{0}$ or on $\partial \Omega_{0}$ (or isolated inclusions), has been deeply studied, see $[17-19,22]$. Let us recall some results in this framework and give formally the construction of the expansion. We consider a fixed domain $\Omega_{0}$ in $\mathbb{R}^{2}$ containing 0 , and another domain $\omega$, centered at the origin 0 . For any $\varepsilon$ small enough, we define the perturbed domain $\Omega_{\varepsilon}$, see Figure 1 , as

$$
\Omega_{\varepsilon}=\Omega_{0} \backslash \omega_{\varepsilon}, \quad \text { with } \omega_{\varepsilon}=\varepsilon \omega .
$$

The problem we are interested in reads 


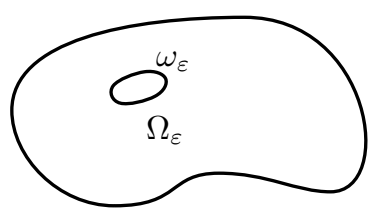

Figure 1. One small interior inclusion.

$$
\left\{\begin{array}{rll}
-\Delta u_{\varepsilon}= & \text { in } \Omega_{\varepsilon} \\
u_{\varepsilon}=0 & \text { on } \partial \Omega_{0} \\
\partial_{\mathbf{n}} u_{\varepsilon}=0 & \text { on } \partial \omega_{\varepsilon}
\end{array}\right.
$$

for some $f \in \mathrm{L}^{2}\left(\Omega_{0}\right)$ whose support does not contain the origin 0 .

Let $u_{0}$ denote the solution of the Dirichlet-Laplacian on $\Omega_{0}$, that is the unperturbed problem:

$$
\left\{\begin{array}{rll}
-\Delta u_{0}= & \text { in } \Omega_{0} \\
u_{0}= & 0 & \text { on } \partial \Omega_{0}
\end{array}\right.
$$

We can estimate $r_{\varepsilon}^{0}:=u_{\varepsilon}-u_{0}$ and so prove that $u_{0}$ is the first order term of the expansion of $u_{\varepsilon}$. Let us explain formally how we determine the following terms. To determine the second one, we notice that $r_{\varepsilon}^{0}$ satisfies:

$$
\left\{\begin{aligned}
-\Delta r_{\varepsilon}^{0} & =0 & & \text { in } \Omega_{\varepsilon}, \\
r_{\varepsilon}^{0} & =0 & & \text { on } \partial \Omega_{0}, \\
\partial_{\mathbf{n}} r_{\varepsilon}^{0} & =-\partial_{\mathbf{n}} u_{0} & & \text { on } \partial \omega_{\varepsilon},
\end{aligned}\right.
$$

where $\mathbf{n}$ denotes the unit normal vector pointing into $\omega$. To catch the following term of the expansion, we lift the Neumann conditions on $\partial \omega_{\varepsilon}$. At first order, we approximate $\partial_{\mathbf{n}} u_{0}$ on $\partial \omega_{\varepsilon}$ by its Taylor expansion at 0 and only lift the condition $\partial_{\mathbf{n}} r_{\varepsilon}^{0}=-\nabla u_{0}(0) \cdot \mathbf{n}$ on $\partial \omega_{\varepsilon}$. The techniques used here rely on the notion of profile, a normalized solution of the Laplace equation in the exterior domain obtained by blow-up of the perturbation. Let us recall here a classical result about the existence and behavior of the profile (see [3]):

Proposition 1.1. Let $\omega$ be a smooth bounded domain of $\mathbb{R}^{2}$ with $0 \in \omega$. We assume that $g \in \mathrm{H}^{-1 / 2}(\partial \omega)$ satisfies $\langle g, 1\rangle_{\mathrm{H}^{-1 / 2} \times \mathrm{H}^{1 / 2}}=0$. Then the boundary value problem

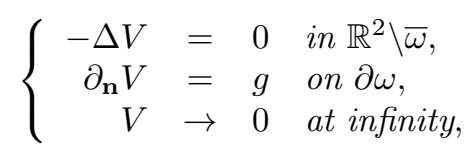

admits a unique weak solution $V_{0}$ in the variational space

$$
\left\{V ; \nabla V \in \mathrm{L}^{2}\left(\mathbb{R}^{2} \backslash \bar{\omega}\right) \text { and } \frac{V}{(1+|X|) \log (2+|X|)} \in \mathrm{L}^{2}\left(\mathbb{R}^{2} \backslash \bar{\omega}\right)\right\} .
$$

Furthermore, this solution can be decomposed as

$$
V_{0}(X)=\sum_{k=1}^{n} V_{0, k}(X)+\mathcal{O}_{\infty}\left(|X|^{-n+1}\right),
$$

where $V_{0, k}$ is an homogeneous harmonic function of order $-k$ and $V_{0, k} \in \mathcal{O}_{\infty}\left(|X|^{-k}\right)$, that is to say $V_{0, k} \in$ $\mathrm{L}\left(\mathbb{R}^{2} \backslash \bar{\omega}\right)$ and for any multi-indice $i \in \mathbb{N}^{2}$, there exists a positive constant $C_{i, k}$ such that

$$
|X|^{k+|i|}\left|\partial^{i} V_{0, k}(X)\right| \leq C_{i, k}, \quad \forall X \in \mathbb{R}^{2} \backslash \bar{\omega} .
$$


The profile $V_{0}$ defined by (1.3) with $g=-\nabla u_{0}(0) \cdot \mathbf{n}$ is used in a fast variable to describe the local behavior of the solution in the perturbed domain. Convergence of the asymptotic expansion is obtained thanks to the decay of the profile at infinity. Thus the expansion of $u_{\varepsilon}$ takes the form

$$
u_{\varepsilon}(x)=u_{0}(x)+\varepsilon V_{0}\left(\frac{x}{\varepsilon}\right)+r_{\varepsilon}^{1}(x), \quad \text { with }\left\|r_{\varepsilon}^{1}\right\|_{\mathrm{H}^{1}\left(\Omega_{\varepsilon}\right)}=\mathcal{O}\left(\varepsilon^{2}\right) .
$$

To explicit the term in $\varepsilon^{2}$, we look at the system satisfied by $r_{\varepsilon}^{1}$ :

$$
\left\{\begin{aligned}
-\Delta r_{\varepsilon}^{1} & =0 & & \text { in } \Omega_{\varepsilon} \\
r_{\varepsilon}^{1} & =-\varepsilon V_{0}(\dot{\bar{\varepsilon}}) & & \text { on } \partial \Omega_{0} \\
\partial_{\mathbf{n}} r_{\varepsilon}^{1} & =\psi_{\varepsilon}^{0} & & \text { on } \partial \omega_{\varepsilon}
\end{aligned}\right.
$$

where $\psi_{\varepsilon}^{0}$ comes from the Taylor expansion of $\partial_{\mathbf{n}} u_{0}$ around 0 . Two terms have to be lifted: one in slow variable to fulfil the Dirichlet condition on $\partial \Omega_{0}$, one in fast variable to satisfy the Neumann condition on the inclusion. For the first one, the model problem writes:

$$
\left\{\begin{array}{rll}
-\Delta w_{1} & =0 & \text { in } \Omega_{0} \\
w_{1} & =\varphi_{1} & \text { on } \partial \Omega_{0}
\end{array}\right.
$$

with $\varphi_{1}$ deduced from the behavior of $V_{0}$. For the term in fast variable, we are in the framework of Proposition 1.1 where $g$ comes from the Taylor expansion of $u_{0}$ and the trace of $w_{1}$. By so on, we have a complete expansion of $u_{\varepsilon}$.

Theorem 1.2. For any $N \in \mathbb{N}$, the solution $u_{\varepsilon}$ of (1.1) reads

$$
u_{\varepsilon}(x)=u_{0}(x)+\sum_{i=0}^{N} \varepsilon^{i+1} V_{i}\left(\frac{x}{\varepsilon}\right)+\sum_{i=1}^{N} \varepsilon^{i+1} w_{i}(x)+\mathcal{O}_{\mathrm{H}^{1}\left(\Omega_{\varepsilon}\right)}\left(\varepsilon^{N+2}\right) .
$$

In this expansion,

- the profiles $V_{i}$, in fast variables, compensate the trace on the inclusion of the $i$-th term in the Taylor expansion of $u_{0}$ and the trace of $w_{j}$ for $j \leq i$,

- the profiles $w_{i}$, in slow variable, compensate the trace of $V_{j}$ for $j<i$ on $\partial \Omega_{0}$.

Remark 1.3. When the inclusion lies on the boundary, the analysis can be more complicated. We refer to $[17,18]$ for more details.

\subsection{Two inclusions case}

We now consider the case of two distinct inclusions inside the domain $\Omega_{0}$. Let $\omega^{-}$, and $\omega^{+}$be two bounded domains of $\mathbb{R}^{2}$, each containing the origin 0 . For $\varepsilon>0$, small enough, we define the perturbed domain $\Omega_{\varepsilon}$ as

$$
\Omega_{\varepsilon}=\Omega_{0} \backslash \overline{\left(\omega_{\varepsilon}^{-} \cup \omega_{\varepsilon}^{+}\right)}, \quad \text { with } \omega_{\varepsilon}^{ \pm}=x_{\varepsilon}^{ \pm}+\varepsilon \omega^{ \pm},
$$

where $x_{\varepsilon}^{ \pm}= \pm \eta_{\varepsilon} \mathbf{d}$ for a given unitary vector $\mathbf{d}$, and a real number $\eta_{\varepsilon}$. Shortly, $\Omega_{\varepsilon}$ consists of $\Omega_{0}$ from which two $\varepsilon$-inclusions at distance $2 \eta_{\varepsilon}$ have been removed, cf. Figure 2 .

We aim at building an asymptotic expansion of the solution $u_{\varepsilon}$ of the Laplace problem in $\Omega_{\varepsilon}$

$$
\left\{\begin{array}{rll}
-\Delta u_{\varepsilon}=f & \text { in } \Omega_{\varepsilon} \\
u_{\varepsilon}=0 & \text { on } \partial \Omega_{0} \\
\partial_{\mathbf{n}} u_{\varepsilon}= & \text { on } \partial \omega_{\varepsilon}^{ \pm}
\end{array}\right.
$$

Again the support of the datum $f$ does not reach the origin 0 . 


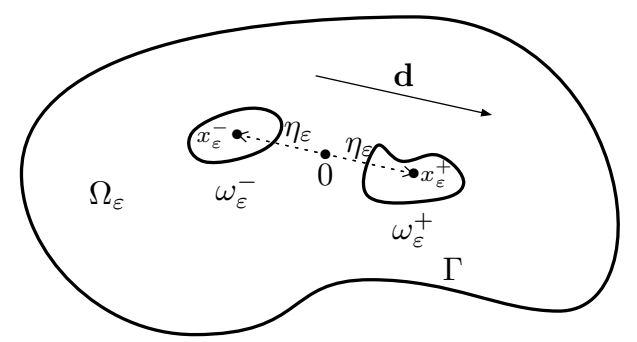

Figure 2. Two inclusions of size $\varepsilon$, at distance $2 \eta_{\varepsilon}$.

The results concerning the case of one inclusion can be easily extended to the case of two (or finitely many) inclusions within two situations:

- Inclusions at distance $\mathcal{O}(1)$. It corresponds to $\eta_{\varepsilon}=\eta$ independent of $\varepsilon$. In this case, the centers $x^{ \pm}$are independent of $\varepsilon$. The decaying profiles $V_{0}^{ \pm}$are harmonic in $\mathbb{R}^{2} \backslash \overline{\omega^{ \pm}}$and satisfy the boundary conditions

$$
\partial_{\mathbf{n}} V_{0}^{ \pm}=-\nabla u_{0}\left(x^{ \pm}\right) \cdot \mathbf{n} \quad \text { on } \partial \omega^{ \pm} .
$$

At first order, the holes do not interact with each other, their contributions are merely superposed

$$
u_{\varepsilon}(x)=u_{0}(x)+\varepsilon\left[V_{0}^{+}\left(\frac{x-x^{+}}{\varepsilon}\right)+V_{0}^{-}\left(\frac{x-x^{-}}{\varepsilon}\right)\right]+r_{\varepsilon}^{1}(x), \quad \text { with }\left\|r_{\varepsilon}^{1}\right\|_{\mathrm{H}^{1}\left(\Omega_{\varepsilon}\right)}=\mathcal{O}\left(\varepsilon^{2}\right) .
$$

- Inclusions at distance $\mathcal{O}(\varepsilon)$. It corresponds to $\eta_{\varepsilon}=c \varepsilon$ with a constant $c \in \mathbb{R}^{+}$. Here the two inclusions constitute a unique pattern at the scale $\varepsilon$. This case is actually handled as a single inclusion $\omega=\omega^{+} \cup \omega^{-}$, selfsimilar with respect to the origin 0 . The expansion reads

$$
u_{\varepsilon}(x)=u_{0}(x)+\varepsilon W_{0}\left(\frac{x}{\varepsilon}\right)+r_{\varepsilon}^{1}(x), \quad \text { with }\left\|r_{\varepsilon}^{1}\right\|_{\mathrm{H}^{1}\left(\Omega_{\varepsilon}\right)}=\mathcal{O}\left(\varepsilon^{2}\right),
$$

where the profile $W_{0}$ is associated with the whole pattern $\omega$.

These two situations show radically different behaviors: no interaction and full interaction. We focus now (see also [9] for more details) on the intermediate cases, where the inclusions are moderately close, as illustrated in Figure 2: the distance between the two inclusions equals $\varepsilon^{\alpha}$ with $\alpha \in(0,1)$. The limit case $\alpha=0$ corresponds to inclusions at distance $\mathcal{O}(1)$ while the other limit $\alpha=1$ corresponds to inclusions at distance $\mathcal{O}(\varepsilon)$. We proceed exactly as for the case of one inclusion. We consider $r_{\varepsilon}^{0}:=u_{\varepsilon}-u_{0}$ which satisfies:

$$
\left\{\begin{aligned}
-\Delta r_{\varepsilon}^{0} & =0 & & \text { in } \Omega_{\varepsilon}, \\
r_{\varepsilon}^{0} & =0 & & \text { on } \partial \Omega_{0}, \\
\partial_{\mathbf{n}} r_{\varepsilon}^{0} & =-\partial_{\mathbf{n}} u_{0} & & \text { on } \partial \omega_{\varepsilon}^{+} \cup \partial \omega_{\varepsilon}^{-} .
\end{aligned}\right.
$$

We introduce the profiles $V_{0}^{ \pm}$defined by (1.3) with $\omega=\omega^{ \pm}$and write:

$$
u_{\varepsilon}(x)=u_{0}(x)+\varepsilon\left[V_{0}^{-}\left(\frac{x-x_{\varepsilon}^{-}}{\varepsilon}\right)+V_{0}^{+}\left(\frac{x-x_{\varepsilon}^{+}}{\varepsilon}\right)\right]+r_{\varepsilon}^{1}(x) .
$$

We check that $r_{\varepsilon}^{1}$ satisfies

$$
\left\{\begin{aligned}
-\Delta r_{\varepsilon}^{1} & =0 & & \text { in } \Omega_{\varepsilon}, \\
r_{\varepsilon}^{1}(x) & =-\varepsilon\left[V_{0}^{-}\left(\frac{x-x_{\varepsilon}^{-}}{\varepsilon}\right)+V_{0}^{+}\left(\frac{x-x_{\varepsilon}^{+}}{\varepsilon}\right)\right] & & \text { for } x \in \partial \Omega_{0}, \\
\partial_{\mathbf{n}} r_{\varepsilon}^{1}(x) & =\mathbf{n} \cdot \nabla u_{0}(0)-\mathbf{n} \cdot \nabla u_{0}(x)-\mathbf{n} \cdot \nabla V_{0}^{-}\left(\frac{x-x_{\varepsilon}^{-}}{\varepsilon}\right) & & \text { for } x \in \partial \omega_{\varepsilon}^{+}, \\
\partial_{\mathbf{n}} r_{\varepsilon}^{1}(x) & =\mathbf{n} \cdot \nabla u_{0}(0)-\mathbf{n} \cdot \nabla u_{0}(x)-\mathbf{n} \cdot \nabla V_{0}^{+}\left(\frac{x-x_{\varepsilon}^{+}}{\varepsilon}\right) & & \text { for } x \in \partial \omega_{\varepsilon}^{-}
\end{aligned}\right.
$$


To determine the order of the remainder, we need more information about the behavior of the trace of $r_{\varepsilon}^{1}$ on the boundaries. Using Proposition 1.1, we know the behavior of $V_{0}^{ \pm}$. Consequently,

- when $x \in \partial \Omega_{0},\left|x-x_{\varepsilon}^{ \pm}\right|$has an expansion into powers of $\varepsilon^{\alpha}$ and then there exist $f_{j, k}$ such that we can rewrite

$$
r_{\varepsilon}^{1}(x)=\sum_{\substack{j \geq 1, k \geq 0, j+\alpha k \leq N}} \varepsilon^{j+\alpha k} f_{j, k}(x)+\mathcal{o}\left(\varepsilon^{N}\right), \quad \forall x \in \partial \Omega_{0} .
$$

- when $x= \pm \varepsilon^{\alpha} \mathbf{d}+\varepsilon X \in \partial \omega_{\varepsilon}^{ \pm},\left|x-x_{\varepsilon}^{ \pm}\right|$admits an expansion into powers of $\varepsilon^{1-\alpha}$ and then we deduce

$$
\partial_{\mathbf{n}} r_{\varepsilon}^{1}(x)=\sum_{\substack{j \geq 0, k \geq 0, 0<j+\alpha k \leq N}} \varepsilon^{j+\alpha k} g_{j, k}^{ \pm}(X)+\sum_{2 \leq j \leq \frac{N}{1-\alpha}} \varepsilon^{j(1-\alpha)} h_{j}^{\mp}(X)+\mathcal{o}\left(\varepsilon^{N}\right)
$$

where the functions $g_{j, k}^{ \pm}$come from the Taylor expansion of $u_{0}$ and $h_{j}^{\mp}$ from the trace of the profiles $V_{0}^{\mp}$. These functions satisfy the compatibility conditions

$$
\int_{\partial \omega^{ \pm}} g_{j, k}^{ \pm}(X) d \sigma_{X}=0 \quad \text { and } \quad \int_{\partial \omega^{ \pm}} h_{j}^{\mp}(X) d \sigma_{X}=0 .
$$

At this step, we have to lift each boundary condition appearing in (1.12) and (1.13). The functions $f_{j, k}$ introduced in (1.12) generate correctors $F_{j, k}$ defined by

$$
\left\{\begin{array}{rlll}
-\Delta F_{j, k} & =0 & & \text { in } \Omega_{0} \\
F_{j, k} & =-f_{j, k} & & \text { on } \partial \Omega_{0}
\end{array}\right.
$$

These correctors do not satisfy the Neumann condition on the boundary of the inclusions $\partial \omega_{\varepsilon}^{ \pm}$and so generate errors on these boundaries. The functions $g_{j, k}^{ \pm}$and $h_{j}^{\mp}$ generate profiles $G_{j, k}^{ \pm}$and $H_{j}^{\mp}$ with same behavior as the first corrector. These profiles satisfy:

$$
\left\{\begin{array} { r l l } 
{ - \Delta G _ { j , k } ^ { \pm } } & { = 0 } & { \text { in } \mathbb { R } ^ { 2 } \backslash \overline { \omega ^ { \pm } } , } \\
{ \partial _ { \mathbf { n } } G _ { j , k } ^ { \pm } } & { = - g _ { j , k } ^ { \pm } } & { \text { on } \partial \omega ^ { \pm } , } \\
{ G _ { j , k } ^ { \pm } } & { \rightarrow 0 } & { \text { at infinity, } }
\end{array} \quad \left\{\begin{array}{rl}
-\Delta H_{j}^{\mp}=0 & \text { in } \mathbb{R}^{2} \backslash \overline{\omega^{ \pm}}, \\
\partial_{\mathbf{n}} H_{j}^{\mp}=-h_{j}^{\mp} & \text { on } \partial \omega^{ \pm}, \\
H_{j}^{\mp} & \rightarrow 0
\end{array}\right.\right.
$$

Then we can give the following term of the expansion of $u_{\varepsilon}$, iterate the procedure and prove the theorem:

Theorem 1.4. The solution $u_{\varepsilon}$ of problem (1.7) admits the expansion at order $N$

$$
\begin{aligned}
& u_{\varepsilon}(x)=u_{0}(x)+\varepsilon\left[V_{0}^{-}\left(\frac{x-x_{\varepsilon}^{-}}{\varepsilon}\right)+V_{0}^{+}\left(\frac{x-x_{\varepsilon}^{+}}{\varepsilon}\right)\right] \\
& \quad+\sum_{(p, q) \in \mathcal{K}_{N}} \varepsilon^{p+\alpha q}\left(v_{p+\alpha q}(x)+\varepsilon\left[V_{p+\alpha q}^{-}\left(\frac{x-x_{\varepsilon}^{-}}{\varepsilon}\right)+V_{p+\alpha q}^{+}\left(\frac{x-x_{\varepsilon}^{+}}{\varepsilon}\right)\right]\right)+r_{\varepsilon}^{N}(x), \quad \text { with }\left\|r_{\varepsilon}^{N}\right\|_{\mathrm{H}^{1}\left(\Omega_{\varepsilon}\right)}=\mathcal{o}\left(\varepsilon^{N}\right),
\end{aligned}
$$

and

$$
\mathcal{K}_{N}=\left\{(p, q) \in \mathbb{Z}^{2} \mid p \geq 0, q \geq-\frac{3}{2} p+1, q \geq-p \text { and } p+\alpha q \leq N\right\} .
$$

The terms $v_{p+\alpha q}, V_{p+\alpha q}^{ \pm}$are built inductively as explained before.

Remark 1.5. Considering only the first profiles $V_{0}^{ \pm}$, we have the expansion

$$
u_{\varepsilon}(x)=u_{0}(x)+\varepsilon\left[V_{0}^{-}\left(\frac{x-x_{\varepsilon}^{-}}{\varepsilon}\right)+V_{0}^{+}\left(\frac{x-x_{\varepsilon}^{+}}{\varepsilon}\right)\right]+r_{\varepsilon}^{1}(x), \quad \text { with }\left\|r_{\varepsilon}^{1}\right\|_{\mathrm{H}^{1}\left(\Omega_{\varepsilon}\right)}=\mathcal{O}\left(\varepsilon^{\min (1+\alpha, 3-2 \alpha)}\right)
$$


We may understand expansion (1.17) in the following way: the main contribution of the two inclusions is merely the superposition of their individual effects. The remainder $r_{\varepsilon}^{1}$ contains information about higher-order influence. It is interesting to describe further the structure of this remainder:

- for $\alpha<2 / 3$, the inclusions are relatively far away from each other. The leading term in $r_{\varepsilon}^{1}$ is $\mathcal{O}\left(\varepsilon^{1+\alpha}\right)$ and arises from the Taylor expansion of $u_{0}$ at the origin 0 ;

- for $2 / 3<\alpha<1$, the inclusions are closer. The remainder $r_{\varepsilon}^{1}$ is $\mathcal{O}\left(\varepsilon^{3-2 \alpha}\right)$ and mainly consists in the interaction between the profiles $V_{0}^{-}$and $V_{0}^{+}$;

- for $\alpha=2 / 3$, the two contributions are equally balanced.

Remark 1.6. In the previous discussion, we restricted to twodimensional domains and homogeneous Neumann boundary conditions on $\partial \omega_{\varepsilon}^{ \pm}$, although generalizations to other conditions are possible. In particular, our results easily extend to Neumann boundary conditions in dimension $d \geq 2$ and Dirichlet conditions in dimension $d \geq 3$. The Dirichlet case in dimension 2 raises non trivial difficulties due to the increasing logarithmic potential.

Besides, one of the inclusions may be localized at the boundary $\Gamma$ of $\Omega_{0}$ (or even simply be removed, the remaining inclusion moving towards the external boundary). The origin does not belong to the domains themselves anymore, but only to their closure (see [9]).

\section{Extension to LinEAR ELASTICITY}

In this section, we state the asymptotic result for the linear elasticity equations. The procedure used for the Laplacian still holds for the Navier equations. Such an analysis is useful in the framework of mechanical engineering for elastic structures with small inhomogeneities, when considering their behavior till rupture. Our aim is to design a numerical strategy dealing with a coarse discretization of the unperturbed domain and able to perform the analysis of the structural response from the elastic phase to complete failure. To that purpose, we consider two macroscopic models dedicated to each of the two phases of the behavior:

- the asymptotic analysis is used to evaluate the influence of the presence of micro-defects on the solution,

- the strong discontinuity approach allows taking into account, at the structural scale, the development of localization zones or cohesive cracks, see [14].

In this paper, we only present results concerning the first phase for the evaluation of stress concentration due to the presence of geometrical defects. We refer to $[6,12,13]$ for a more complete presentation.

We evaluate the influence of geometrical perturbations by a multiscale asymptotic analysis of the equations of linear elasticity. The inclusions are assumed to be located on the boundary.

Let $\Omega_{0}$ be a domain of $\mathbb{R}^{2}$ such that the regular point 0 belongs to the boundary and we assume that the boundary coincides with the abscissa axis in a small neighbourhood of 0 . The case of a single perturbation was presented in [13]. We present the case of two relatively close inclusions here (see [6] for more details). We consider a domain $\Omega_{\varepsilon}$ pierced with two perturbations of size $\varepsilon$ near 0 :

$$
\Omega_{\varepsilon}=\Omega_{0} \backslash \overline{\omega_{\varepsilon}^{1} \cup \omega_{\varepsilon}^{2}}, \quad \text { with } \quad \omega_{\varepsilon}^{j}=x_{\varepsilon}^{j}+\varepsilon \omega^{j}, \quad x_{\varepsilon}^{1}=\varepsilon^{\alpha} \mathbf{d} \text { and } x_{\varepsilon}^{2}=-\varepsilon^{\alpha} \mathbf{d}, \quad \alpha \in(0,1) .
$$

We assume that $\omega^{j}$ contains 0 and $\mathbf{d}$ is the tangent vector of the boundary on 0 . We denote by $\mathbf{H}_{\infty}^{j}$ the unbounded domains obtained by a blow-up around each perturbation:

$$
\mathbf{H}_{\infty}^{j}=\mathbb{R} \times \mathbb{R}_{+} \backslash \overline{\omega^{j}} .
$$

The problem we focus on is written on the perturbed domain as:

$$
\left\{\begin{array}{rlll}
-\mu \Delta \mathbf{u}_{\varepsilon}-(\lambda+\mu) \nabla \operatorname{div} \mathbf{u}_{\varepsilon} & =\mathbf{0} & & \text { in } \Omega_{\varepsilon} \\
\mathbf{u}_{\varepsilon} & =\mathbf{u}^{d} & & \text { on } \Gamma_{d} \\
\sigma\left(\mathbf{u}_{\varepsilon}\right) \cdot \mathbf{n} & =\mathbf{g} & & \text { on } \Gamma_{n}
\end{array}\right.
$$


where $\Gamma_{d}$ and $\Gamma_{n}$ denote the Dirichlet and Neumann boundary of the domain respectively, $\Gamma_{n}$ includes the boundary of the perturbation and $\mathbf{g}$ is supposed to be zero in a neighborhood of the perturbation. In the former equation, $\mathbf{u}_{\varepsilon}$ denotes the displacement and $\sigma$ stands for the stress tensor:

$$
\sigma_{i j}(\mathbf{u})=\lambda\left(\partial_{1} \mathbf{u}_{1}+\partial_{2} \mathbf{u}_{2}\right) \delta_{i j}+\mu\left(\partial_{i} \mathbf{u}_{j}+\partial_{j} \mathbf{u}_{i}\right) .
$$

The solution of (2.1) is given at first order by

$$
\mathbf{u}_{\varepsilon}(x)=\mathbf{u}_{0}(x)-\varepsilon \sum_{j=1}^{2}\left[\alpha_{1} \mathbf{v}_{1}^{j}\left(\frac{x-x_{\varepsilon}^{j}}{\varepsilon}\right)+\alpha_{2} \mathbf{v}_{2}^{j}\left(\frac{x-x_{\varepsilon}^{j}}{\varepsilon}\right)\right]+\mathcal{O}\left(\varepsilon^{\min (1+\alpha, 3-2 \alpha)}\right),
$$

with $\mathbf{u}_{0}$ the solution on the unperturbed domain, $\alpha_{1}=\sigma_{11}\left(\mathbf{u}_{0}\right)(\mathbf{0})$ and $\alpha_{2}=\sigma_{12}\left(\mathbf{u}_{0}\right)(\mathbf{0})$. The profiles $\mathbf{v}_{1}^{j}$ and $\mathbf{v}_{2}^{j}$ are obtained as solution of an homogeneous Navier equation stated on the unbounded domain $\mathbf{H}_{\infty}^{j}$ with Neumann boundary conditions on the boundary of the normalized perturbation:

$$
\left\{\begin{array}{rlll}
-\mu \Delta \mathbf{v}_{\ell}^{j}-(\lambda+\mu) \nabla \operatorname{div} \mathbf{v}_{\ell}^{j} & =\mathbf{0} & & \text { in } \mathbf{H}_{\infty}^{j} \\
\sigma\left(\mathbf{v}_{\ell}^{j}\right) \cdot \mathbf{n}^{j} & =\mathrm{G}_{\ell}^{j} & \text { on } \partial \mathbf{H}_{\infty}^{j} \\
\mathbf{v}_{\ell}^{j} & \rightarrow & 0 & \text { at infinity }
\end{array}\right.
$$

with $\mathbf{G}_{1}^{j}=\left(\mathbf{n}_{1}^{j}, 0\right), \mathbf{G}_{2}^{j}=\left(0, \mathbf{n}_{1}^{j}\right)$ and $\mathbf{n}_{1}^{j}$ the first component of the outer normal to $\partial \mathbf{H}_{\infty}^{j}$.

\section{Numerical COMPUtATions}

\subsection{Superposition method}

The computation of the solution $u_{\varepsilon}$ of problem (1.7) (or in a same way of (2.1)) is not straightforward since a very fine mesh is required if $\varepsilon$ is small. For such values of $\varepsilon$, it is natural to use the asymptotic expansion. Precisely, we approximate $u_{\varepsilon}$ by its first order expansion

$$
u_{1}(x)=u_{0}(x)+\varepsilon\left[V_{0}^{-}\left(\frac{x-x_{\varepsilon}^{-}}{\varepsilon}\right)+V_{0}^{+}\left(\frac{x-x_{\varepsilon}^{+}}{\varepsilon}\right)\right] .
$$

This means that $u_{0}$ and the profiles $V_{0}^{ \pm}$have to be computed. While $u_{0}$ is the solution of a classical boundary value problem (in an $\varepsilon$-independent domain which may be coarsely meshed), the profiles are solution of a problem posed on an infinite domain. The computation of the profiles in a reference geometry has been done (once and for all if the shape of the defects is prescribed) by a finite element computation in a bounded domain with an approximate boundary condition on an artificial boundary (see next section).

In the context of fracture mechanics, we aim at predicting the behavior of damage structures till rupture under the presence of geometrical defects in the material. The asymptotic expansion (2.2) is used to compute the displacement field without meshing to the scale of the small holes or inhomogeneities. The superposition formula (2.2) is not necessarily suited for implementation in an existing finite element code. Indeed, we have preferred a kinematic enrichment of the approximation space through partition of unity, see [16,23].

The displacement field discretisation is then of the form:

$$
\mathbf{u}^{h}(x)=\mathbf{u}_{0}^{h}(x)-\varepsilon \sum_{j=1}^{2} \sum_{\ell=1}^{2} \sum_{i \in \mathcal{J}_{j}} N^{i}(x)\left[\boldsymbol{\alpha}_{i \ell, 1}^{j} \tilde{\mathrm{v}}_{\ell, 1}^{j}\left(\frac{x-x_{\varepsilon}^{j}}{\varepsilon}\right)+\boldsymbol{\alpha}_{i \ell, 2}^{j} \tilde{\mathrm{V}}_{\ell, 2}^{j}\left(\frac{x-x_{\varepsilon}^{j}}{\varepsilon}\right)\right],
$$

where $\mathcal{J}_{j}$ denotes the indices of the nodes located in the enrichment zone related to perturbation $j . N^{i}$ are the standard shape functions associated to node $i$ and $\boldsymbol{\alpha}_{i \ell, k}^{j}$ is a two-component vector consisting of the degrees of freedom related to the enrichment function $\tilde{\mathrm{v}}_{\ell, k}^{j}$, obtained as approximations of the profiles $\mathbf{v}_{\ell}^{j}$.

A master-slave strategy has been used to keep stability of the problem to be solved (see [6] for further details). 


\subsection{Computation of the profiles}

The problem defining the profiles is set in an unbounded domain. We use articifial boundary conditions to obtain an accurate approximation of the profiles. The numerical results shown hereafter have been performed with the Finite Element Library MÉLINA, see [21].

\section{Laplace equation.}

Let us first consider the computation of profiles satisfying (1.3) in the case of the Laplacian. In order to compute the profiles $V_{0}^{ \pm}$involved in formula (3.1), we introduce the normalized vectorial profile $\mathbf{V}=\mathbf{V}_{\omega}$, solution of the following exterior boundary value problem

$$
\left\{\begin{array}{rlll}
-\Delta \mathbf{V} & = & 0 & \text { in } \mathbb{R}^{2} \backslash \omega \\
\partial_{\mathbf{n}} \mathbf{V}=\mathbf{g} & \text { on } \partial \omega \\
\mathbf{V} & \rightarrow 0 & \text { at infinity }
\end{array}\right.
$$

with $\mathbf{g}=-\mathbf{n}$. We can recover $V_{0}^{ \pm}$from $\mathbf{V}_{\omega^{ \pm}}$via the formula $V_{0}^{ \pm}=\nabla u_{0}(0) \cdot \mathbf{V}_{\omega^{ \pm}}$, so that formula (3.1) reads

$$
u_{1}(x)=u_{0}(x)+\varepsilon \nabla u_{0}(0) \cdot\left[\mathbf{V}_{\omega^{-}}\left(\frac{x-x_{\varepsilon}^{-}}{\varepsilon}\right)+\mathbf{V}_{\omega^{+}}\left(\frac{x-x_{\varepsilon}^{+}}{\varepsilon}\right)\right] .
$$

The profile $\mathbf{V}$ will be approximated componentwise: $V$ and $g$ denote the first component of $\mathbf{V}$ and $\mathbf{g}$, respectively (of course, the same can be done for the second component). Several approaches are available to compute $V$ : integral equation, infinite elements, truncated domain with integral representation or artificial boundary condition. To determine an artificial boundary condition, we seek a linear combination of $V$ and its normal and tangent derivatives for which the expansion into powers of $R$ at infinity starts with $R^{-p}$ for various value of the order $p$. For $p=0,1,2$, we obtain Dirichlet, Robin, and Ventcel conditions respectively:

$$
\begin{aligned}
& V=0 \\
& V+R \partial_{\mathbf{n}} V=0, \\
& V+\frac{3 R}{2} \partial_{\mathbf{n}} V-\frac{R^{2}}{2} \Delta_{\tau} V=0 .
\end{aligned}
$$

The considered problem is then

$$
\left\{\begin{array}{cl}
-\Delta V=0 & \text { in } B(0, R) \backslash \omega \\
\partial_{\mathbf{n}} V=g & \text { on } \partial \omega \\
(3.4) \text { or }(3.5) \text { or }(3.6) & \text { on } \partial B(0, R) .
\end{array}\right.
$$

Figure 3 gives the convergence for these three conditions as $R$ goes to infinity in the model case of ring with internal radius 1 and external radius $R$. The domain is meshed into 64 square elements of degree $\mathbb{Q}_{8}$, a nonisoparametric interpolation $\mathbb{Q}_{10}$ is performed. We compare the solution of (3.7) to a reference computation and observe the slopes 1, 2, 3 as expected. We notice that the Ventcel condition (3.6) is not satisfactory when $R$ becomes large, due to the unaccurate approximation of the tangential derivative (this could be improved with a finer mesh).

\section{Navier equations.}

We now deal with the computation of the profiles defined by (2.3) associated with the Navier equation. As in the case of the Laplace operator, we use a artificial boundary condition to compute accurately the profiles. The easiest artificial condition is the Dirichlet one. Cancelling the leading singular parts at infinity of the solution 


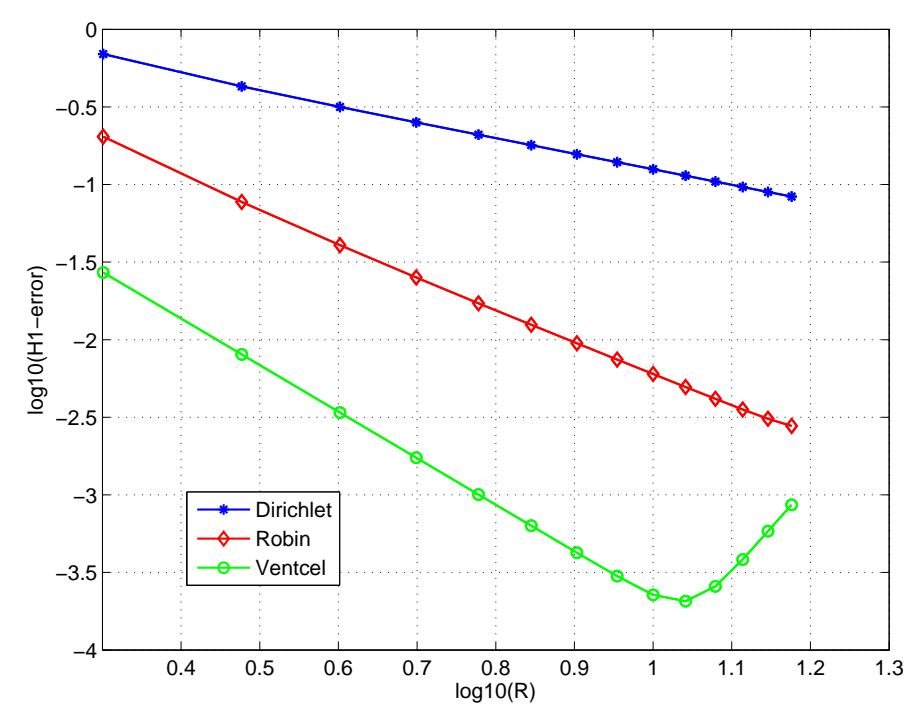

FIGURE 3. Convergence of the approximate problems (3.7) with a fixed number of DOF.

leads to a more accurate boundary condition (see $[5,7,10])$

$$
\sigma(\mathbf{u}) \mathbf{n}+\frac{1}{R} \frac{E}{1+\nu}\left[\begin{array}{cc}
\frac{1}{1-\nu} & 0 \\
0 & 1
\end{array}\right] \mathbf{u}+\frac{1}{R} \frac{E(1-\nu)}{2(1+\nu)(1-2 \nu)}\left[\begin{array}{ll}
0 & 0 \\
0 & 1
\end{array}\right] \Delta_{\tau} \mathbf{u}=0,
$$

set on the circle of radius $R$. The physical parameters $E$ and $\nu$ are such that the quantity in front of the Laplace-Beltrami operator is nonnegative: Young's modulus $E$ is nonnegative and Poisson's coefficient $\nu$ takes values in the interval $(-1,0.5)$. We have the following relations between the coefficients:

$$
\lambda=\frac{\nu E}{(1+\nu)(1-2 \nu)}, \quad \mu=\frac{E}{2(1+\nu)} .
$$

The problem (3.8) is degenerate and we have to prove the existence of solution. To this purpose, we study in [8] the case of the Laplace operator as a model problem. We focus on the Ventcel boundary value problem for Laplace operator such as

$$
\left\{\begin{array}{rlll}
-\Delta u & = & & \text { in } \Omega \\
\partial_{n} u+\alpha u+\beta \Delta_{\tau} u & = & \varphi & \text { on } \partial \Omega
\end{array}\right.
$$

under the unusual condition $\beta>0$. We reformulate the boundary value problem into a nonlocal equation on the boundary $\partial \Omega$. For this, we introduce the Dirichlet-to-Neumann map $\Lambda$ associated to the Laplace operator on $\Omega$ : this operator is defined from $\mathrm{H}^{1 / 2}(\partial \Omega)$ onto $\mathrm{H}^{-1 / 2}(\partial \Omega)$ by $\Lambda(\psi)=\partial_{n} U$ where $U$ is the solution of the boundary value problem

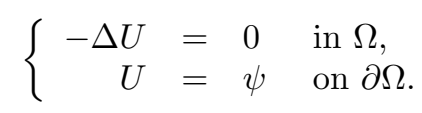

The introduction of the Dirichlet-to-Neumann map allows us to rewrite (3.9) as the surface equation

$$
\beta \Delta_{\tau} w+\Lambda w+\alpha w=\varphi \text { on } \partial \Omega .
$$

Pseudodifferential and spectral techniques lead to existence and uniqueness results apart from exceptional cases. Then, we consider perforated geometries and give conditions to remove the genericity restriction.

We expect these arguments to generalize for condition (3.8). 


\subsection{Numerics}

\section{Laplace equation}

Let us present numerical simulations for the Laplace problem (1.7). To compare $u_{\varepsilon}$ and its 0-th and first order approximations, we need to compute $u_{\varepsilon}$ accurately. Figure 4 shows the differences $u_{\varepsilon}-u_{0}$ and $u_{\varepsilon}-u_{1}$ on the example of two ellipses. The value $\varepsilon=0.0585$ is relatively large for visibility reasons, but nevertheless the approximation given by the first order approximation $u_{1}$ is much better than $u_{0}$. The principal error in $u_{\varepsilon}-u_{0}$ is mainly concentrated around the holes, it is partially corrected in $u_{\varepsilon}-u_{1}$. In Figure 5 , we present the errors

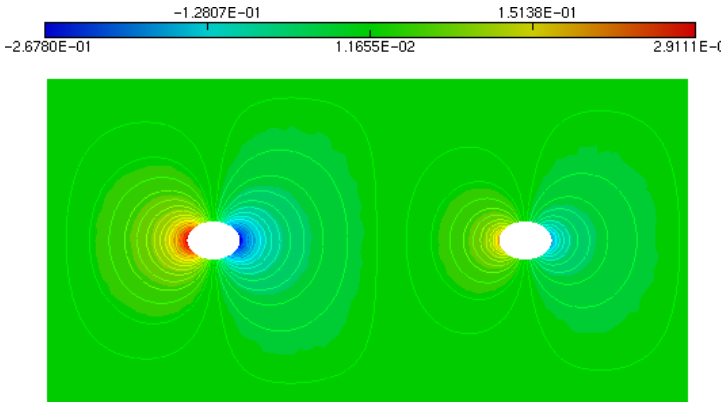

(a) $u_{\varepsilon}-u_{0}$.

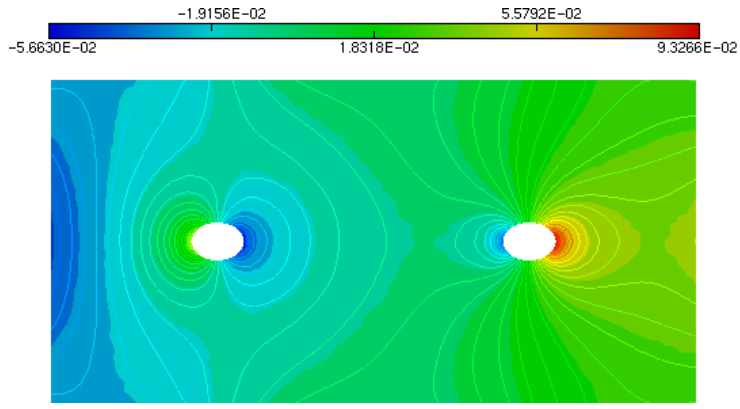

(b) $u_{\varepsilon}-u_{1}$.

FigURE 4. $u_{\varepsilon}-u_{0}$ and $u_{\varepsilon}-u_{1}$ for $\varepsilon=0.0585$ and $\alpha=0.5$.

(in the $\mathrm{H}^{1}\left(\Omega_{\varepsilon}\right)$-norm) obtained for the two approximations $u_{0}$ and $u_{1}$ in the case where the two inclusions are ellipses, and $\alpha=0.2$. When we compute the local convergence rates as the slopes between two consecutive points in Figure 5, we recover the expected rate $1+\alpha=1.2$ for $u_{\varepsilon}-u_{1}$, cf. expansion (1.17).

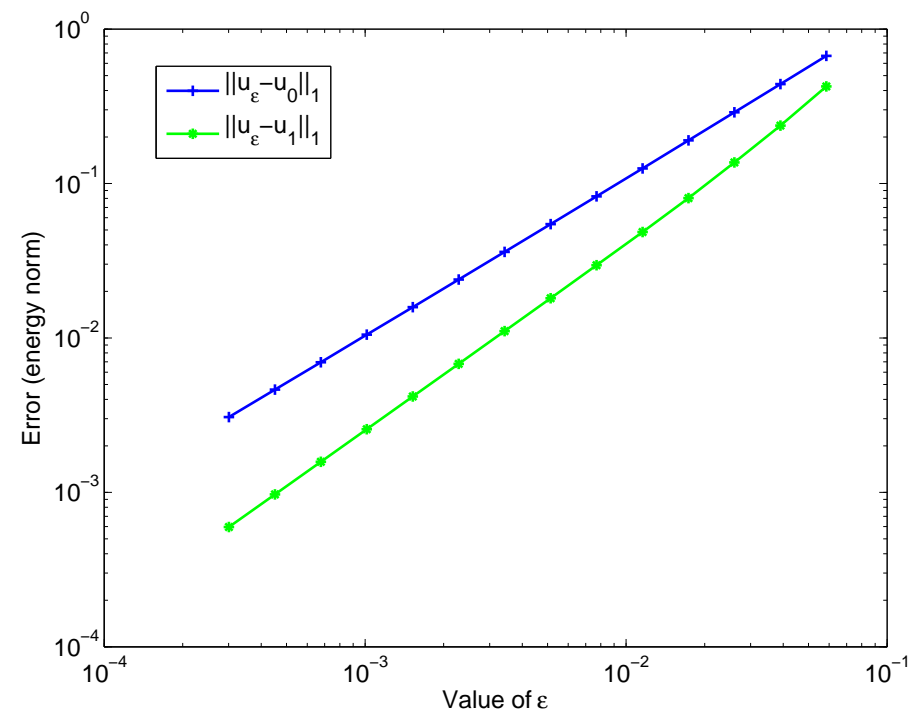

FIGURE 5. Energy norms of $u_{\varepsilon}-u_{0}$ and $u_{\varepsilon}-u_{1}$ for $\alpha=0.2$. 


\section{Navier equation}

We present now the results obtained considering a domain submitted to a tensile load (Figure 6(a)). The domain is pierced by two perturbations: the first one is centered at point $O_{1}=(105,0)$ and is of radius 2 mm and the second of radius $1.5 \mathrm{~mm}$ is centered at point $\mathrm{O}_{2}=(135,0)$.

In order to validate the enrichment strategy adopted for the description of the influence of small defects, we compare the results obtained in terms of displacement and stress fields for a so called reference computation carried out on a fine discretization of the real geometry (Figure 6(b)) by standard Finite Element approach, and an enriched computation performed on a coarse discretization (Figure 6(c)) of the unperturbed domain.

Figure 7 gives the obtained results in terms of the displacement field in the direction of the traction for both discretization and interpolations. The relative error between those two computations is lower than $0.25 \%$ allowing to conclude that the proposed strategy gives satisfactory results.

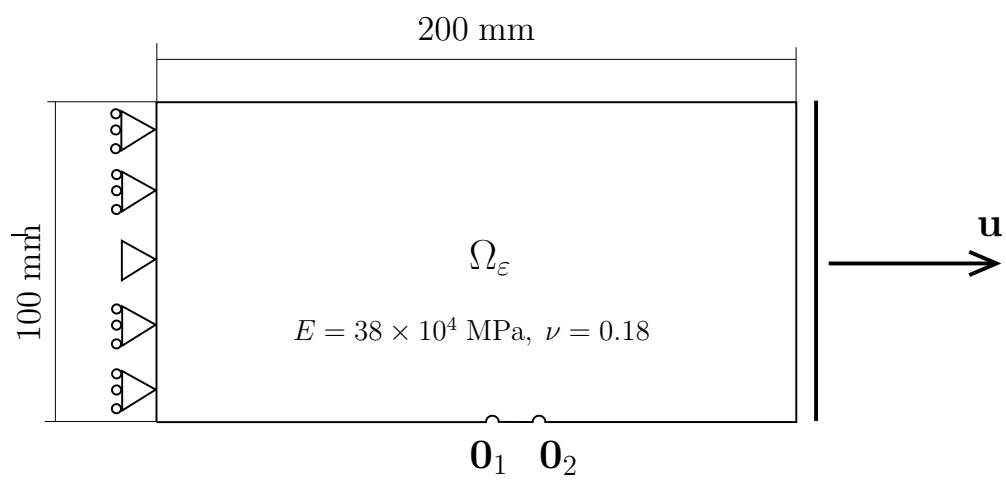

(a) Problem definition: geometry, loading and material properties

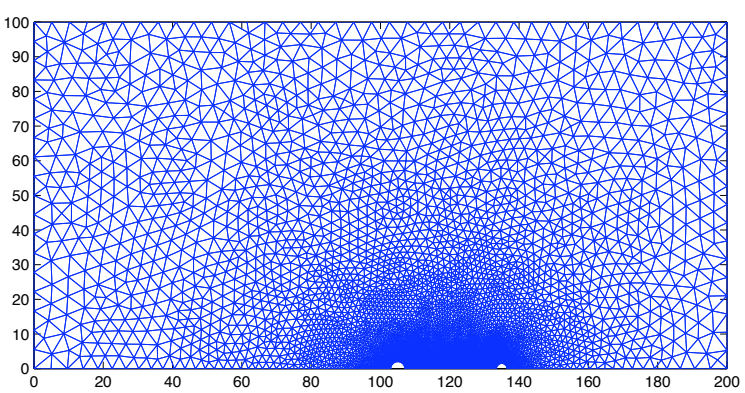

(b) Fine discretization of the domain (reference computation)

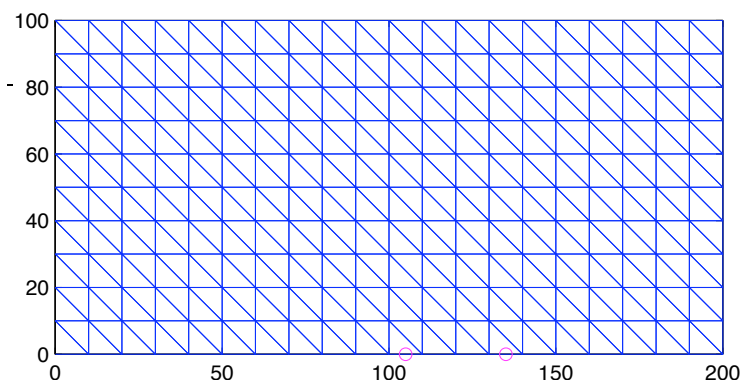

(c) Coarse mesh for computation of $\mathbf{u}_{\varepsilon}^{h}$

FiguRE 6. Problem definition: geometry, loading, material properties and discretizations

\section{REFERENCES}

[1] H. Ammari, E. Bonnetier, Y. CapdeboscQ, M. Tanter, M. Fink. Electrical impedance tomography by elastic deformation. SIAM J. Appl. Math. 68(6) (2008) 1557-1573.

[2] H. Ammari, H. Kang, M. Lim, H. Zribi. Layer potential techniques in spectral analysis. I. Complete asymptotic expansions for eigenvalues of the Laplacian in domains with small inclusions. Trans. Amer. Math. Soc. 362(6) (2010) $2901-2922$.

[3] C. Amrouche, V. Girault, J. Giroire. Dirichlet and Neumann exterior problems for the $n$-dimensional Laplace operator: an approach in weighted Sobolev spaces. J. Math. Pures Appl. (9) 76(1) (1997) 55-81.

[4] M. F. Ben Hassen, E. Bonnetier. An asymptotic formula for the voltage potential in a perturbed $\varepsilon$-periodic composite medium containing misplaced inclusions of size E. Proc. Roy. Soc. Edinburgh Sect. A 136(4) (2006) 669-700. 
[5] V. Bonnaillie-Noël, D. Brancherie, M. Dambrine, G. Vial. Artificial boundary conditions to compute correctors in linear elasticity. Submitted (2011).

[6] V. Bonnaillie-Nö̈l, D. Brancherie, M. Dambrine, S. Tordeux, G. Vial. Effect of micro-defects on structure failure : coupling asymptotic analysis and strong discontinuity approach. Eur. Journal Comput. Mech. 19(1-3) (2010) 165-175.

[7] V. Bonnaillie-Nö̈L, M. Dambrine, F. Hérau, G. Vial. Absorbing conditions for the linear elasticity equations. In preparation (2011).

[8] V. Bonnaillie-Nö̈L, M. Dambrine, F. Hérau, G. Vial. On generalized Ventcel's type boundary conditions for Laplace operator in a bounded domain. SIAM J. Math. Anal. 42(2) (2010) 931-945.

[9] V. Bonnaillie-NoËl, M. Dambrine, S. Tordeux, G. Vial. Interactions between moderately close inclusions for the Laplace equation. Math. Models Methods Appl. Sci. 19(10) (2009) 1853-1882.

[10] V. Bonnaillie-Nö̈l, M. Dambrine, G. Vial. Small defects in mechanics. AIP Conference Proceedings ICNAAM 20111389 (2011) 1416-1424.

[11] E. Bonnetier, M. Vogelius. An elliptic regularity result for a composite medium with "touching" fibers of circular crosssection. SIAM J. Math. Anal. 31(3) (2000) 651-677 (electronic).

[12] D. Brancherie, M. Dambrine, G. Vial, P. Villon. Ultimate load computation, effect of surfacic defect and adaptative techniques. In 7th World Congress in Computational Mechanics, Los Angeles 2006.

[13] D. Brancherie, M. Dambrine, G. Vial, P. Villon. Effect of surface defects on structure failure: a two-scale approach. Eur. Journal Comput. Mech. 17(5-7) (2008) 613-624.

[14] D. Brancherie, A. Ibrahimbegović. Novel anisotropic continuum-discrete damage model capable of representing localized failure of massive structures. Part I: theoretical formulation and numerical implementation. Engineering Computations 26(1-2) (2009) 100-127.

[15] Y. CapdeboscQ, M. Vogelius. A general representation formula for boundary voltage perturbations caused by internal conductivity inhomogeneities of low volume fraction. M2AN 37(1) (jan 2003) 159-173.

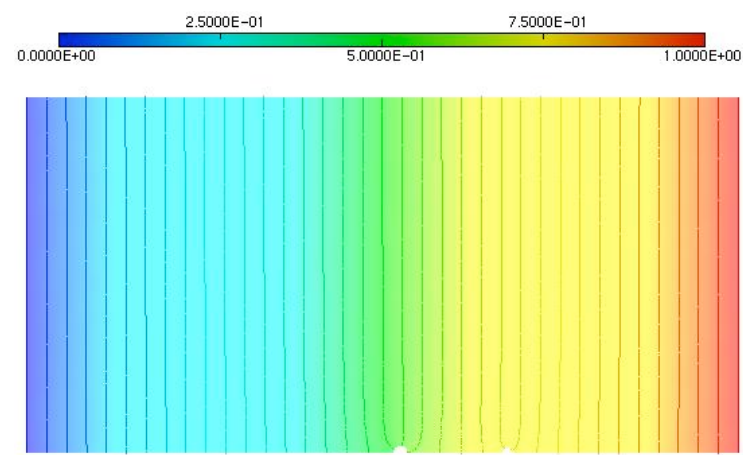

(a) Reference computation on the fine discretization

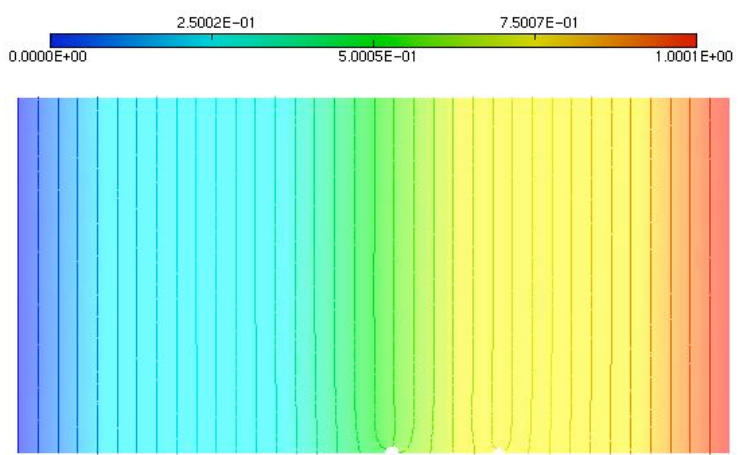

(b) Computation with kinematic enrichment on the coarse discretization

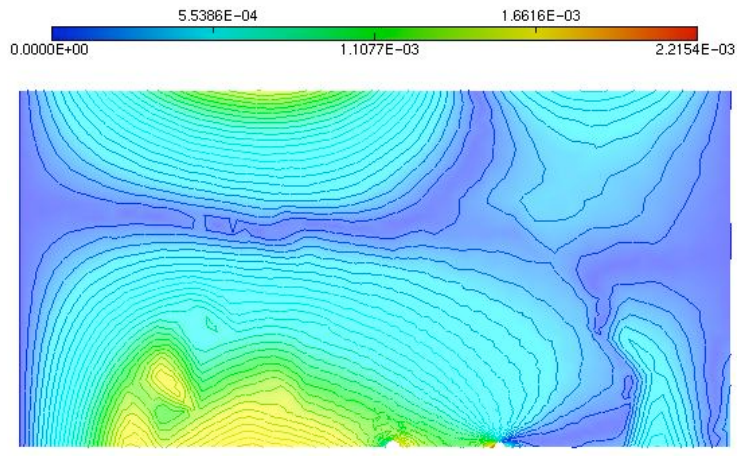

(c) Relative error

FiguRE 7. Displacement field $u_{x}$ obtained by standard and enriched computation, relative error map 
[16] E. Chahine, P. Laborde, J. Pommier, Y. Renard, M. Salaün. Study of some optimal XFEM type methods. In Advances in meshfree techniques, volume 5 of Comput. Methods Appl. Sci., pages 27-38. Springer, Dordrecht 2007.

[17] M. Dambrine, G. Vial. Influence of a boundary perforation on the Dirichlet energy. Control Cybernet. 34(1) (2005) 117-136.

[18] M. Dambrine, G. Vial. A multiscale correction method for local singular perturbations of the boundary. M2AN Math. Model. Numer. Anal. 41(1) (2007) 111-127.

[19] A. M. IL'IN. Matching of asymptotic expansions of solutions of boundary value problems, volume 102 of Translations of Mathematical Monographs. American Mathematical Society, Providence, RI 1992.

[20] T. Lewiński, J. SokoŁowski. Topological derivative for nucleation of non-circular voids. The Neumann problem. In Differential geometric methods in the control of partial differential equations (Boulder, CO, 1999), volume 268 of Contemp. Math., pages 341-361. Amer. Math. Soc., Providence, RI 2000.

[21] D. Martin. MÉLinA, bibliothèque de calculs éléments finis. http://perso.univ-rennes1.fr/daniel.martin/melina (2008).

[22] V. Maz'ya, S. Nazarov, B. PlamenevskiJ. Asymptotic theory of elliptic boundary value problems in singularly perturbed domains. Vol. I, volume 111 of Operator Theory: Advances and Applications. Birkhäuser Verlag, Basel 2000.

[23] J. M. Melenk, I. BabušKa. The partition of unity finite element method: basic theory and applications. Comput. Methods Appl. Mech. Engrg. 139(1-4) (1996) 289-314.

[24] S. A. Nazarov, J. SokoŁowski. Asymptotic analysis of shape functionals. J. Math. Pures Appl. (9) 82(2) (2003) $125-196$.

[25] M. S. Vogelius, D. Volkov. Asymptotic formulas for perturbations in the electromagnetic fields due to the presence of inhomogeneities of small diameter. M2AN Math. Model. Numer. Anal. 34(4) (2000) 723-748. 